\title{
Foodborne Infections and Intoxications in Hyderabad India
}

\author{
R. V. Sudershan, ${ }^{1}$ R. Naveen Kumar, ${ }^{1}$ L. Kashinath, ${ }^{1}$ V. Bhaskar, ${ }^{2}$ and K. Polasa ${ }^{1}$ \\ ${ }^{1}$ Food and Drug Toxicology Research Center, National Institute of Nutrition, Indian Council of Medical Research (ICMR), \\ Jamai-Osmania, Tarnaka, Hyderabad 500007, India \\ ${ }^{2}$ Statistical Division, National Institute of Nutrition, Indian Council of Medical Research (ICMR), Hyderabad 500007, India
}

Correspondence should be addressed to R. V. Sudershan; vemulasr@yahoo.com

Received 22 October 2013; Revised 23 December 2013; Accepted 6 January 2014; Published 13 February 2014

Academic Editor: Suminori Kono

Copyright (c) 2014 R. V. Sudershan et al. This is an open access article distributed under the Creative Commons Attribution License, which permits unrestricted use, distribution, and reproduction in any medium, provided the original work is properly cited.

Foodborne diseases are one of the health hazards and causes of morbidity and mortality in developing countries. In India there are no systematic studies to understand the types of foods involved and the etiological agent causing the disease. Therefore, a pilot study was proposed to investigate the food poisoning cases, undertaken by the Ronald Ross Institute of Tropical Diseases, which is a referral hospital for foodborne diseases in Hyderabad. Food and stool/rectal swabs of the patients affected were collected for microbiological examination. Odds ratio and 95\% confidence interval were used to express the statistical significance of the differences. Epidemiological, environmental, and laboratory components indicated that Staphylococcus aureus was the etiological agent in most of the cases and in one case Salmonella spp. were the main cause of food poisoning. This study indicated the need to take up foodborne disease surveillance under the Indian context and to identify the common high-risk food commodities for microbial contamination and identification.

\section{Introduction}

Foodborne diseases cause morbidity and mortality in the general population and they have emerged as a growing public health and economic problem in many countries during the last 2 decades. Foodborne disease can be defined as any disease of an infectious or toxic nature caused by or thought to be caused by the consumption of food or water [1]. The global burden of foodborne disease is currently unknown but the World Health Organization (WHO) has responded to this data gap by launching a new initiative to provide better estimates. In 2005 it was reported that 1.8 million people died from diarrheal diseases largely due to contaminated food and water [2]. The scientific investigations/reports on outbreak of foodborne diseases in India for the past 29 (1980-2009) years indicated that a total of 37 outbreaks involving 3,485 persons have been affected due to food poisoning [3]. The estimated 47.8 million cases of foodborne diseases, resulting in 127,839 hospitalizations and 3037 deaths, transmitted through food each year in the United States of America (USA) alone [4].

In the United States, incidence of foodborne illness is documented through FoodNet, a reporting system used by public health agencies that capture foodborne illness in over $13 \%$ of the population. Of the 10 pathogens tracked by FoodNet, Salmonella, Campylobacter, and Shigella are responsible for most cases of foodborne illnesses. When both the estimated number of cases and mortality rate are considered for bacterial, viral, and parasitic cases of foodborne illness, Salmonella causes $31 \%$ of food related deaths, followed by Listeria (28\%), Campylobacter (5\%), and Escherichia coli O157:H7 (3\%) [5].

Foodborne diseases are being investigated to prevent both ongoing transmissions of disease and similar outbreaks in the future. The importance of foodborne disease investigation includes control of ongoing outbreaks, detection and removal of implicated foods, identification of specific risk factors related to the host, the agent, and the environment, identification of factors that contribute to contamination, growth, survival, and dissemination of the suspected agent, prevention of future outbreaks and strengthening of food safety policies, acquisition of epidemiological data for risk assessment of foodborne pathogens, and stimulation of research that will help in the prevention of similar outbreaks [6].

In India foodborne diseases are not categorized separately in the Health Information of India. For example, in 
the official document of health information, Government of India for 2004, 9575112 cases of acute diarrhoel diseases including gastroenteritis with 2855 deaths have been recorded and cases of foodborne disease may have been categorized under gastroenteritis [7]. Foodborne disease surveillance is essential for estimating the burden of foodborne diseases and monitoring trends, identifying priorities, and setting policy in the control and prevention of foodborne diseases and their outbreaks, detection, and evaluation of control strategies [8].

At present as per Indian Food regulations, medical practitioners should report the occurrence of food poisoning to the food health authorities. State Food Laboratories are supposed to carry out food poisoning investigation. To carry out foodborne disease investigation epidemiological, environmental, and laboratory components should integrate closely at the beginning, during the course, and at the end of an investigation. This type of investigation system is lacking in India. In India there are no systematic studies to understand the types of foods involved and the etiological agent causing the disease. Therefore in order to create awareness among policy makers a pilot study was undertaken to investigate the food poisoning cases, with the support of Ronald Ross Institute of Tropical Diseases, a referral hospital for foodborne diseases in Hyderabad.

\section{Materials and Methods}

The investigation in Hyderabad was carried out after receiving information on food poisoning cases from the Institute of Tropical Diseases, which was a Government Fever Hospital earlier. Once a suspected outbreak of foodborne illness has been reported, a series of steps were taken as follows.

2.1. Receipt of Initial Data. The initial data on outbreak of suspected food poisoning during the period of 2003-05 was received by phone call from the Institute of Tropical Diseases.

2.2. Verification of the Diagnosis. The illness was confirmed by the doctor at the hospital and based on their symptoms and preliminary case history the investigation was done.

\subsection{Determination of Whether an Outbreak Has Occurred.} Initially the information on age, sex, symptoms, and type of food consumed was gathered from the patients at the hospital and also by visiting the affected area where ill persons are located and interviewing few persons and determining that food poisoning has occurred. Additional data was collected by using questionnaire which includes demographic information, occupation or disease setting, time of exposure, signs of symptoms and treatment, time of initial symptom, $74 \mathrm{hr}$ food history, quantity of food taken, additional risk factor information, medical history and laboratory findings, and additional case reports.

2.4. Description of Cases in terms of Time, Place, and Person. The information on time of exposure and the time of onset of illness was collected and the incubation period was calculated directly. Assessment by place provided the information on the geographical extent of the outbreak and also provided important clues about its cause. Person characteristics were used to identify features that are common to cases as a clue to etiology or source of infection. Age, sex, ethnicity, and occupation were among the characteristic features that were used to describe the case studies.

2.5. Formulation of Hypothesis. In this stage the data was summarized and formulation of hypothesis was done to explain the outbreak. Based on the time of exposure and the time of onset of illness individual incubation period was calculated. These formed the bases for Hypothesis formulation. The mode and vehicle of transmission as well as the specific exposure that caused the disease were also considered. This hypothesis was strongly supported by epidemiological, laboratory, and food data.

2.6. Analytical, Epidemiological, Environmental, and Laboratory Studies. Analytical epidemiological studies determine a statistical association between certain food items and illness. Association of food items with the incident was analyzed. Odds ratio (OR) and their 95\% confidence intervals were used to express the statistical significance of the differences. For environmental component studies a food preparation review was done to determine if foods involved could have been contaminated or otherwise abused in a manner that would have led to the illness being investigated. This review indicates what happened prior to the consumption of a suspect food that could have resulted in making someone ill.

2.7. Sample Handling and Processing. Stool samples/rectal swabs of the patients and the food samples at the site of outbreak were collected for microbiological examination. Consent was obtained from individual patient before the collection of biological sample. The samples were inoculated into Carry Blair transport medium and brought to the laboratory in an icebox. Aseptic condition was maintained during transportation of the biological samples. The samples were kept in refrigerator until further microbiological analysis. Direct microscopic examination of the samples and culture was carried out to see the kind and number of microorganisms present in them. Twenty-five grams of the food sample was taken in $225 \mathrm{~mL}$ of sterilized buffered peptone water. After thorough mixing of the sample $0.1 \mathrm{~mL}$ of the sample was inoculated on selective media like XLD (Xylose Lysine Deoxycholate Agar) for Salmonella, BPA (Baired Parker Agar) for S. aureus, BCA (Bacillus Cereus Agar) for B. cereus, TCBS (Thiosulfate Citrate Bile Sucrose Agar) for Vibrio spp., and MacConkey Agar for Escherichia coli. For isolation of Salmonella spp. approximately $1 \mathrm{~g}$ or $1 \mathrm{~mL}$ of each sample was transferred to selenite F broth (qualitative enrichment) and incubated at $37^{\circ} \mathrm{C}$ for $24 \mathrm{~h}$. The enrichments were streaked on xylose lysine desoxycholate agar and incubated for $24 \mathrm{~h}$ at $37^{\circ} \mathrm{C}$. Stool samples/rectal swabs were inoculated on these selective media to get pure culture. After incubation at $37^{\circ} \mathrm{C}$ for $24 \mathrm{hrs}$ the colonies were observed and the identification of pure culture from food and stool samples was done by studying colony characteristics, microscopy, motility test, 
TABle 1: Age and sex distribution of recorded cases of food poisoning during 2003-2005.

\begin{tabular}{|c|c|c|c|c|}
\hline Age group (years) & Male & Female & Total & Percentage \\
\hline $0-4$ & - & - & - & - \\
\hline $5-9$ & 4 & 2 & 6 & 7.4 \\
\hline $10-14$ & 8 & 8 & 16 & 19.7 \\
\hline $15-19$ & 7 & 7 & 14 & 17.2 \\
\hline $20-29$ & 8 & 12 & 20 & 24.6 \\
\hline $30-39$ & 5 & 4 & 9 & 11.1 \\
\hline $40-49$ & 2 & 6 & 8 & 9.8 \\
\hline More than 50 & 5 & 3 & 8 & 9.8 \\
\hline Total & 39 & 42 & 81 & - \\
\hline
\end{tabular}

TABLE 2: Number of outbreaks (group size and sporadic cases) during 2003-2005.

\begin{tabular}{|c|c|c|c|c|c|}
\hline Group size & 2003 & 2004 & 2005 & Total outbreaks & Percentage \\
\hline $2-5$ & 1 & - & 3 & 4 & 40 \\
\hline $6-10$ & - & 1 & 1 & 2 & 20 \\
\hline $11-15$ & - & - & - & - & - \\
\hline $16-20$ & - & 3 & - & 3 & 30 \\
\hline More than 20 & - & - & 1 & 1 & 10 \\
\hline Sporadic & - & - & - & - & - \\
\hline Total & 1 & 4 & 4 & 10 & - \\
\hline
\end{tabular}

and biochemical tests such as catalase, oxidase, coagulase, and carbohydrate fermentation tests. Ethical approval was obtained from the National Institute of Nutrition, Hyderabad, the Indian Council of Medical Research, and Ministry of Health and Family Welfare, Govternment of India.

2.8. Statistical Analysis. The statistical analysis was performed using SPSS 10.0 version. An association between intake of food and the presence of foodborne pathogen was found using chi-square analysis. Odds ratio values with 95\% CI were computed to obtain the risk of presence of foodborne pathogen in a particular food. The odds ratio was calculated based on the presence of foodborne pathogen in food pertaining to ill and nonill patients.

\section{Results and Discussions}

The investigated cases of foodborne diseases in Hyderabad were 10 outbreaks involving 996 persons. A total of 81 patients were examined during the study. The patients aged between 5 and 9 are $7 \%, 10$ and 14 were 19\%, 15 and 19 were 17\%, 20 and 29 were $24 \%, 30$ and 39 were $11 \%, 40$ and 49 were $9 \%$, and more than 50 were $9 \%$ (Table 1). Most of the food poisoning affected persons had vomiting and diarrhoea symptoms. The type of food responsible for these outbreaks includes kaddu ka khee (sweet prepared from desiccated milk and bottle guard), milkshake, chicken biryani (dish prepared from rice and chicken), fruit salad, colostrum, mango juice, and jaggery rice and the etiological agent in most of the cases was $S$. aureus and in one case Salmonella species was isolated.

Food preparation review indicated that all the incriminated foods collected during food poisoning outbreaks were contaminated before consumption. During the investigation period the majority of the outbreaks (40\%) involved group of 2-5 persons while $20 \%$ involved group of $6-10$ persons (Table 2). Larger outbreak was involved in more than 15 persons (40\%). Out of 10 cases investigated 9 were having incubation period ranging from 2 to $6 \mathrm{hrs}$ which is typical of $S$. aureus food poisoning. The latent period of $S$. aureus is 30 mins $-8 \mathrm{hrs}$; the latent period and symptoms of $S$. aureus were matching with most of the investigated food poisoning cases.

A total of 14 food samples and 12 rectal swab/stool samples were collected during the study period. Food and stool samples were subjected to microbiological analysis. Grams staining of the culture revealed the presence of Gram-positive cocci in most of the food and stool samples while in one case Gram-negative bacilli was observed. S. aureus was detected in most of the food and stool samples. The colonies of $S$. aureus in the food samples ranged from 4.0 to $9.5 \times 10^{8} \mathrm{cfu} / \mathrm{g}$ and in the stool $/$ rectal swab samples it was $5-8.5 \times 10^{8} \mathrm{cfu} / \mathrm{g}$. The Salmonella count was $3.0 \times 10^{4} \mathrm{cfu} / \mathrm{g}$ in the food sample and in the stool sample it was $2.5 \times 10^{6} \mathrm{cfu} / \mathrm{g}$ (Table 3 ).

Among all the cases investigated more outbreaks have been observed in marriage functions and at household level. Among the food poisoning cases investigated 7 cases were showing statistically significant association between illness and consumption of implicated food. No significant association was observed between illness and consumption of food in the other 3 cases. Analysis of the food items consumed showed that the odds ratio of food poisoning cases who had kaddu ka kheer was 5-6 times higher than those who did not consume, the $95 \%$ confidence interval being 2.1-11.2. The odds ratio of persons who had fruit salad was 4 times higher, 
TABLE 3: Bacteriological analysis (cfu/g) of samples collected during food poisoning outbreaks in Hyderabad during 2003-2005.

\begin{tabular}{lcc}
\hline Sample & Staphylococcus aureus & Salmonella spp. \\
\hline Kaddu Ka Kheer & $4.5-5.5 \times 10^{8}$ & $\mathrm{ND}$ \\
Fruit salad & $6.5 \times 10^{8}$ & $\mathrm{ND}$ \\
Milk shake & $4.0 \times 10^{8}$ & $\mathrm{ND}$ \\
Chicken biriyani & $8.5-9.5 \times 10^{8}$ & $\mathrm{ND}$ \\
Colostrum & $9.0 \times 10^{8}$ & $\mathrm{ND}$ \\
Mango juice & $\mathrm{ND}$ & $3.0 \times 10^{4}$ \\
Jaggary rice & $4.0 \times 10^{8}$ & $\mathrm{ND}$ \\
\hline
\end{tabular}

ND: not detected.

TABLE 4: Risk estimate of different food samples using odds ratio and $95 \%$ confidence interval.

\begin{tabular}{lccc}
\hline Sl. no & Food & Odds ratio & $95 \%$ CI \\
\hline 1 & Kaddu ka kheer & 5.6 & $2.1-11.2$ \\
2 & Fruit salad & 4 & $1.8-8.5$ \\
3 & Milk shake & 4.5 & $1.9-10.2$ \\
4 & Chicken biriyani & 3.1 & $0.6-154.4$ \\
5 & Colostrum & 10 & $1.4-69.2$ \\
6 & Mango juice & 6 & $0.3-101.5$ \\
7 & Jaggary rice & 6 & $0.3-101.5$ \\
\hline
\end{tabular}

the $95 \%$ confidence interval being 1.8-8.5. The odds ratio of persons who had milk was 4.5 times higher, $95 \%$ confidence interval being 1.9-10.2. The odds ratio of the persons who had chicken biryani was 3 times higher, 95\% confidence interval being 1.5-5.8. The odds ratio of the persons who had colostrum was 10 times higher, the 95\% CI being 1.4-69.2 (Table 4).

It was noticed that kaddu ka kheer consistently appeared to have the highest risk of disease. There is a $95 \%$ chance that increased risk was associated with eating kaddu ka kheer between 2.1 and 11.2 times than for persons who did not eat kaddu ka kheer. Eating colostrum $(\mathrm{OR}=10 ; 95 \% \mathrm{CI}=$ 1.4-69.2) was identified as having a statistically significant association of being ill. These cases were 4 times riskier (95\% CI $=1.8-8.5)$ than normal persons who have consumed fruit salad. In mango juice, jaggery rice, and chicken biryani measures of association (OR) were severalfold greater than one, although there was no statistical significance. Similar type of investigation of a large foodborne outbreak was reported in US; eating cucumber salad (OR $=12.1$; 95\% CI $=2.9-50.5)$ was identified as having statistically significant association with illness [9].

Various studies reported in India indicated the incidences of foodborne diseases, wherein the food implicated were sandwiches [10], soybean milk [11], stale rice [12], chicken [13], bread and vegetable curry, and butter milk [14], where as studies in other countries indicated the incidence of foodborne diseases wherein the foods implicated were Beef [15], Pork meat [16], frozen steaks [17], poultry, and other meat items [18]. Outbreak of food poisoning may be attributed to the type of food being consumed, which is based on the food habits of the people specific to the country problems associated during transport, climatic conditions, food storage, and awareness about food safety concerns.

The places of occurrence of most of the food poisoning cases were home (40\%) and marriage functions (40\%). Eating leftover food was one of the reasons for most of the food poisoning cases. Eating at commercial food establishments was one of the risk factors for sporadic cases of Salmonellosis [19] and Campylobacteriosis [20]. The present study indicated that in 9 out of 10 food poisoning outbreaks the bacteria were found to be $S$. aureus. Earlier individual investigation of foodborne disease in India reported variety of bacteria like Vibrio parahaemolyticus, Vibrio vulnificus, Vibrio fluvialis, Escherichia coli, and Salmonella enteritidis including Staphylococcus aureus. Some of the unusual bacteria like Salmonella weltevreden and Salmonella bornum also have been isolated where as other countries the incidence of foodborne diseases were due to Salmonella typhimurium [21], Salmonella muenchen, Salmonella enteritidis, Escherichia coli 0157:H7, and Campylobacter. Salmonella is one of the most important pathogens responsible for human food poisoning in the developing world and chicken products are widely acknowledged to be a significant reservoir of salmonella [22].

There is a $95 \%$ chance that foodborne disease outbreak in India is occasionally reported in newspapers, mostly from urban areas. The 10 outbreaks investigated during the study period probably represent a small proportion of those, which actually occurred in Hyderabad over this period. Outbreaks were recorded only when the patients are hospitalized. In most of the investigated cases $S$. aureus has been isolated. The high counts indicated the possible etiological role of $S$. aureus in the food poisoning outbreak as counts of more than $10^{3}$ to $10^{4} / \mathrm{g}$ in foods are considered to be contaminated and capable of causing food poisoning outbreak.

Out of the 10 food poisoning outbreaks investigated, only in 7 outbreaks consumption of particular food item and illness were associated significantly and it may be due to wrong perceptions of the affected people and the implicated foods such as mango juice and jaggery rice were not reported earlier. The investigated food poisoning cases could be less due to several reasons like discarding contaminated food, delay in reaching the outbreak site due to delay in communication, delay in analysis due to a failure to alert the lab in advance, and due to early discharge of the patients from the hospital. In addition to health consequences the economic consequences of foodborne disease such as loss of wages, cost of treatment, and loss of affected food articles are being overlooked. The economic cost of Staphylococcus aureus outbreak for 64 persons in Hyderabad was Rs 8.079 [23]. The economic impact of foodborne diseases on an annual basis in Canada, United States, and New Zealand ranges from 1.1 billion to 7 billion $[24,25]$.

\section{Conclusions}

The food poisoning cases investigated during 2003-05 in Hyderabad are an infective and intoxicating type of foodborne diseases caused by Salmonella spp. and Staphylococcus aureus. It is interesting that most of the food poisoning cases 
investigated are caused due to the organism Staphylococcus aureus. To prevent food poisoning outbreaks appropriate educative and preventive measures should be taken up. The health authorities should strengthen considerably the foodborne disease surveillance system and follow it with efficient education and extension activity or various aspects of food safety. The initiatives like epidemiological and laboratory components should be incorporated in our country to conduct appropriate surveillance programmes, so that the real burden of foodborne disease can be determined at various national levels.

\section{Conflict of Interests}

There is no conflict of interests regarding the publication of this paper.

\section{Acknowledgments}

The authors acknowledge the encouragement given during the course of study by the Director, National Institute of Nutrition (ICMR), Hyderabad, India, and also Superintendent, Ronald Ross Institute of Communicable Diseases, Hyderabad, for permitting them to collect the samples and data during the study. The authors also thank the subjects for their participation in the study.

\section{References}

[1] M. R. Adams and M. O. Moss, "Significance of food borne diseases," Food Microbiology, vol. 2, no. 163, pp. 160-164, 2003.

[2] D. G. Newell, M. Koopmans, L. Verhoef et al., "Food-borne diseases-the challenges of 20 years ago still persist while new ones continue to emerge," International Journal of Food Microbiology, vol. 139, supplement 1, pp. S3-S15, 2010.

[3] R. V. Sudershan, R. N. Kumar, and K. Polasa, "Foodborne diseases in India-a review," British Food Journal, vol. 114, no. 5, pp. 661-680, 2012.

[4] CDC, "Estimates of foodborne illness in the United States," 2011, http://www.cdc.gov/foodborneburden/2011-foodborne-estimates.html.

[5] P. S. Mead, L. Slutsker, V. Dietz et al., "Food-related illness and death in the United States," Emerging Infectious Diseases, vol. 5, no. 5, pp. 607-625, 1999.

[6] WHO, "Foodborne disease outbreaks. Guidelines for investigation and control," Tech. Rep., WHO, Geneva, Switzerland, 2007.

[7] Health Information of India. Central Bureau of Health Intelligence, Directorate General of Health Services, Ministry of Health and Family Welfare, Government of India, New Delhi, 2004.

[8] R. V. Bhat, "Safety and quality of processed foods," Indian Food Industry, vol. 23, no. 6, pp. 58-61, 2004.

[9] J. S. Yoder, S. Cesario, V. Plotkin, X. Ma, K. K. Shannon, and M. S. Dworkin, "Outbreak of enterotoxigenic Escherichia coli infection with an unusually long duration of illness," Clinical Infectious Diseases, vol. 42, no. 11, pp. 1513-1517, 2006.

[10] M. K. Lalitha, N. M. Walter, J. Mary, and V. I. Mathan, "An abstract of gastroenteritis due to Vibrio parahaemolyticus in Vellore," The Indian Journal of Medical Research, vol. 78, pp. 611615, 1983.
[11] S. B. Kulshrestha, K. N. Kapoor, S. V. S. Malik, and P. N. Khanna, "Enterotoxigenic Escherichia coli from an outbreak with cholerigenic syndromes of gastroenteritis," The Journal of Communicable Diseases, vol. 21, no. 4, pp. 313-317, 1989.

[12] S. P. Choudhary, K. G. Narayan, S. N. Saxena, M. L. Mago, and P. C. John, "Isolation of Salmonella bornum (6, 7, 14: $\left.\mathrm{Z}_{38}\right)$ for the first time in India," Indian Journal of Medical Sciences, vol. 39, no. 3, pp. 45-46, 1985.

[13] R. J. Thekdi, A. G. Lakhani, V. B. Rale, and M. V. Panse, "An outbreak of food poisoning suspected to be caused by Vibrio fluvialis," Journal of Diarrhoeal Diseases Research, vol. 8, no. 4, pp. 163-165, 1990.

[14] M. Abraham, M. Pai, G. Kang et al., "An outbreak of food poisoning in Tamil Nadu associated with Yersinia enterocolitica," Indian Journal of Medical Research, vol. 106, pp. 465-468, 1997.

[15] J. B. McLaughlin, L. J. Castrodale, M. J. Gardner, R. Ahmed, and B. D. Gessner, "Outbreak of multidrug-resistant Salmonella typhimurium associated with ground beef served at a school potluck," Journal of Food Protection, vol. 69, no. 3, pp. 666-670, 2006.

[16] U. Buchholz, B. Brodhun, S. O. Brockmann et al., "An outbreak of Salmonella München in Germany associated with raw pork meat," Journal of Food Protection, vol. 68, no. 2, pp. 273-276, 2005.

[17] E. S. Laine, J. M. Scheftel, D. J. Boxrud et al., "Outbreak of Escherichi coli O157:H7 infections associated with nonintact blade-tenderized frozen steaks sold by door-to-door vendors," Journal of Food Protection, vol. 68, no. 6, pp. 1198-1202, 2005.

[18] R. J. Meldrum, D. Tucker, R. M. M. Smith, and C. Edwards, "Survey of Salmonella and Campylobacter contamination of whole, raw poultry on retail sale in Wales," Journal of Food Protection, vol. 68, no. 7, pp. 1447-1449, 2005.

[19] J. M. Cowden, D. Lynch, C. A. Joseph et al., "Case-control study of infections with Salmonella enteritidis phage type 4 in England," British Medical Journal, vol. 299, no. 6702, pp. 771773, 1989.

[20] P. Effler, M.-C. Ieong, A. Kimura et al., "Sporadic Campylobacter jejuni infections in Hawaii: associations with prior antibiotic use and commercially prepared chicken," Journal of Infectious Diseases, vol. 183, no. 7, pp. 1152-1155, 2001.

[21] M. Jacck, S. Ellen, P. Dennis et al., "A multistate outbreak of Salmonella enterica serotype Typhimurium infection linked to raw milk consumption," Journal of Food Protection, vol. 67, no. 10, pp. 2165-2170, 2004.

[22] G. Ozbey and H. B. Ertas, "Salmonella spp. isolation from chicken samples and identification by polymerase chain reaction," Bulgarian Journal of Veternary Medicine, vol. 9, no. 1, pp. 67-73, 2006.

[23] P. Sudhakar, R. Nageshwara, R. V. Bhat, and C. P. Gupta, "The economic impact of a foodborne disease outbreak due to Staphylococcus aureus," Journal of Food Protection, vol. 51, no. 11, pp. 898-900, 1988.

[24] E. C. Todd, "Costs of acute bacterial foodborne disease in Canada and the United States," International Journal of Food Microbiology, vol. 9, no. 4, pp. 313-326, 1989.

[25] W. G. Scott, H. M. Sutt, R. J. Lake, and M. G. Baker, "Economic cost to New Zealand of foodborne infectious disease," The New Zealand Medical Journal, vol. 14, pp. 281-284, 2000. 


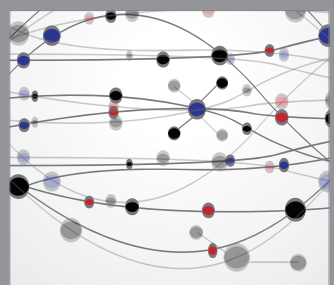

The Scientific World Journal
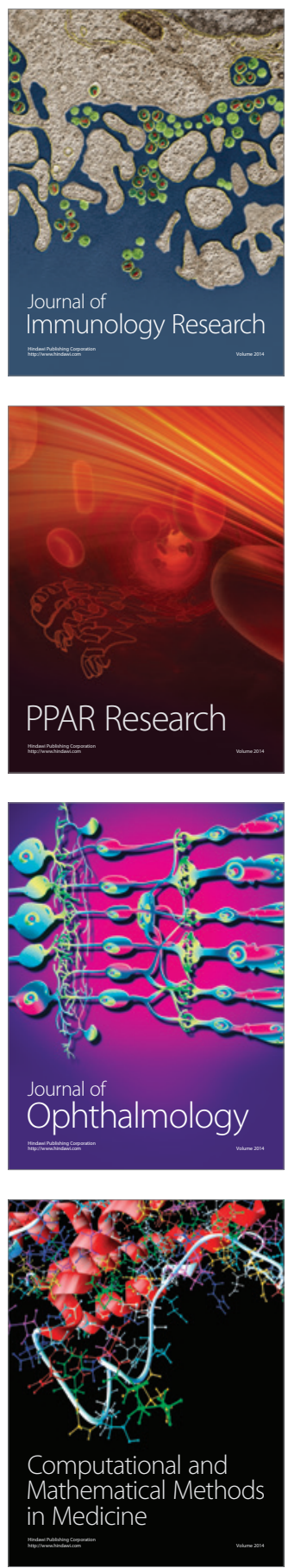

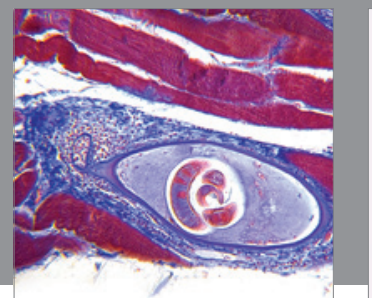

Gastroenterology

Research and Practice
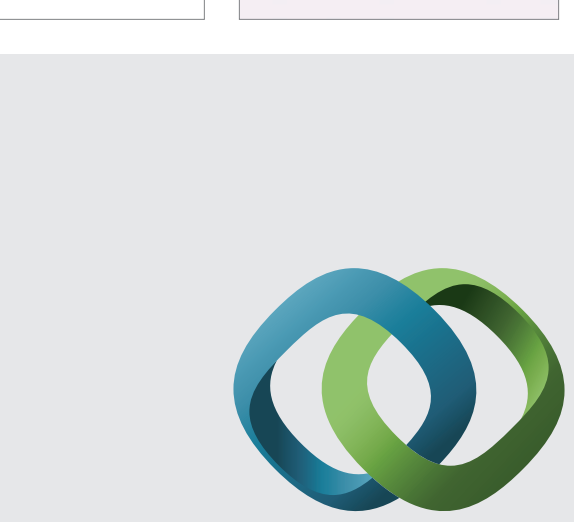

\section{Hindawi}

Submit your manuscripts at

http://www.hindawi.com
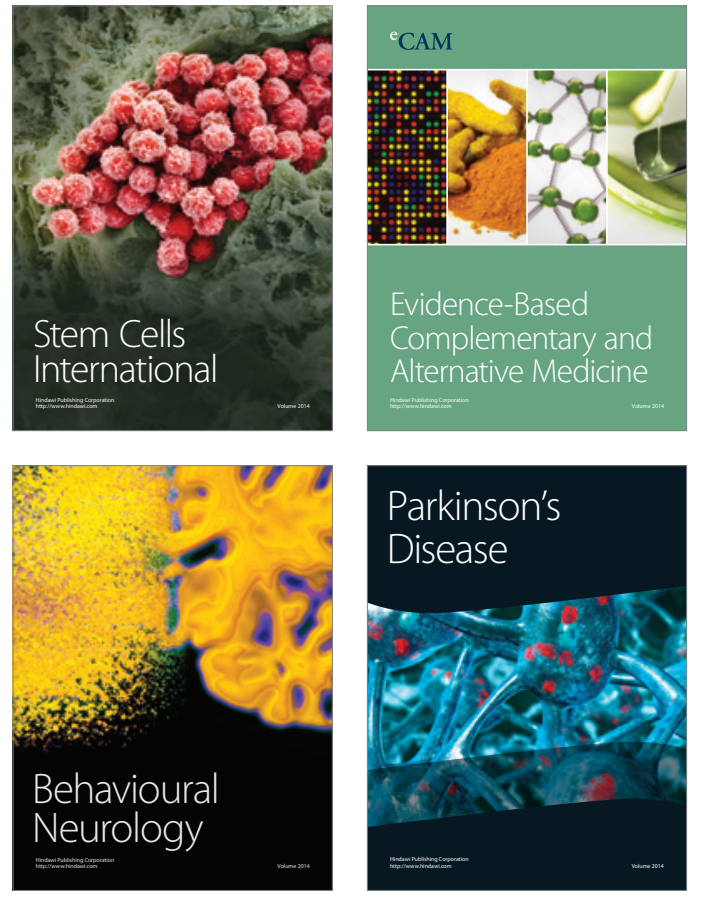
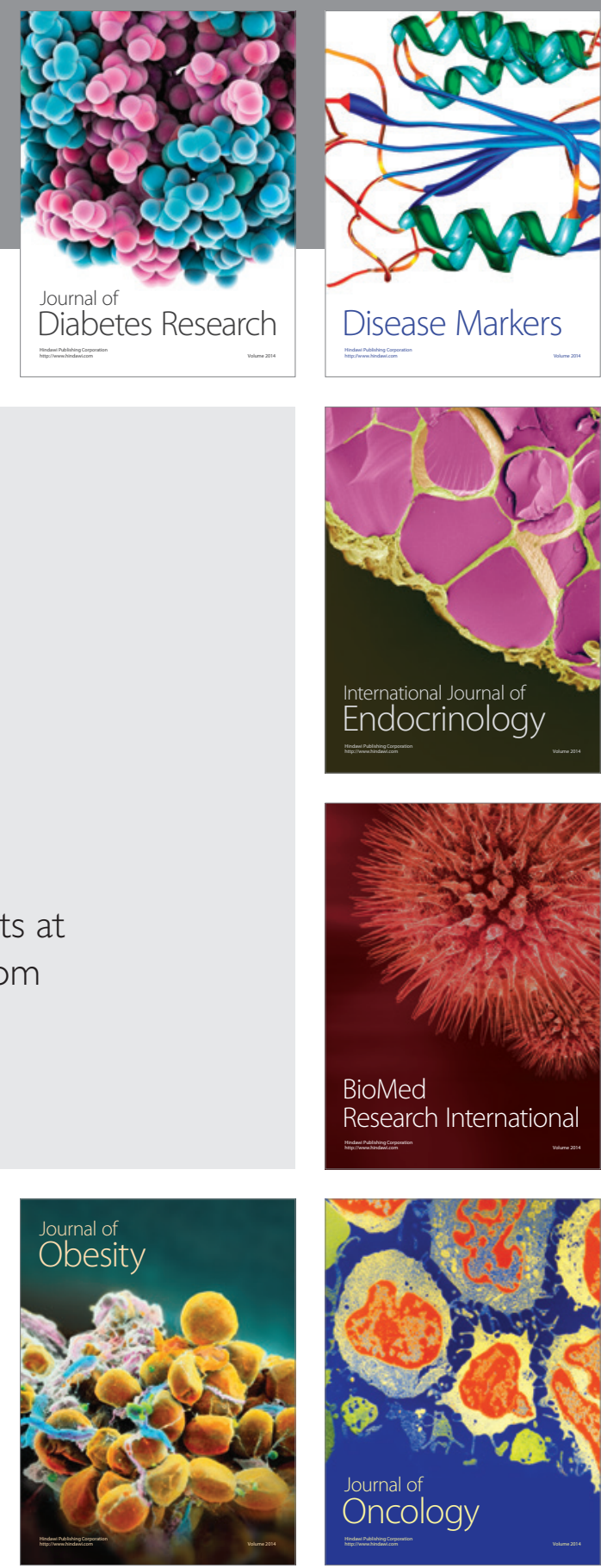

Disease Markers
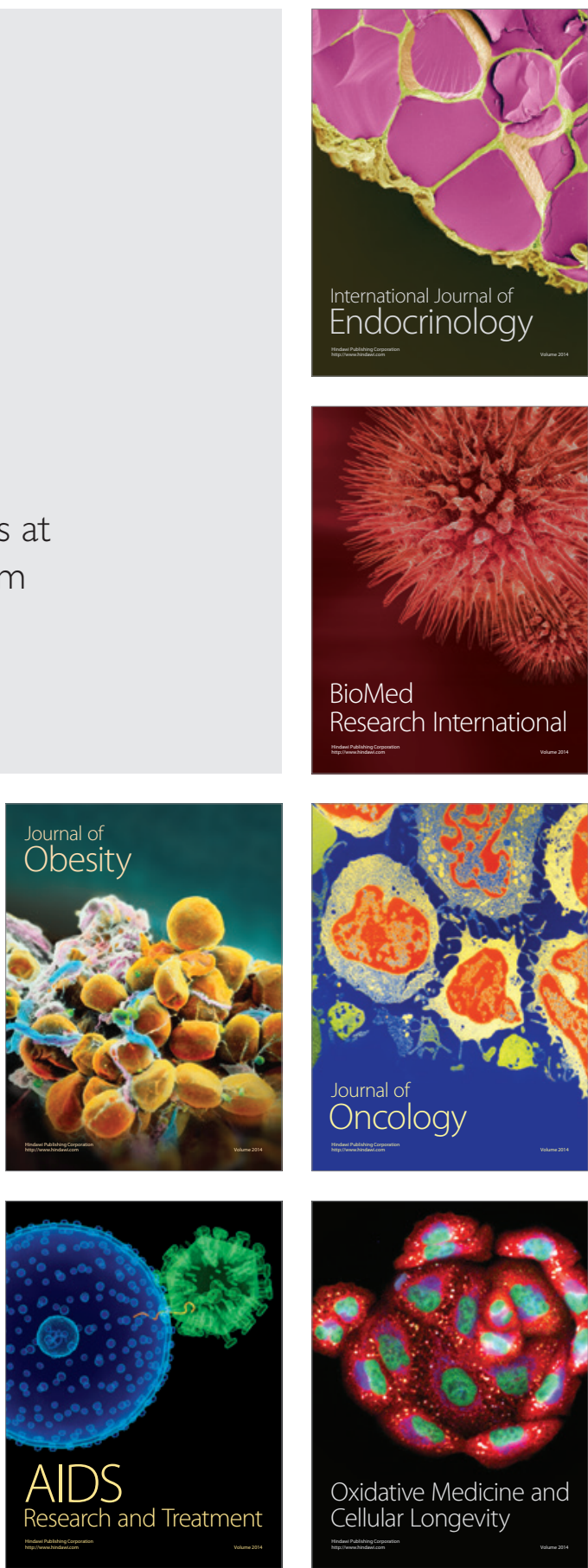